\title{
PARENTALIDADES EN FAMILIAS DIVERSAS
}

\section{PARENTING IN DIVERSE FAMILIES}

\section{Tatiana Blanco Álvarez*}

\author{
RESUMEN
}

El concepto de familia se encuentra en una importante transición; ya no se considera únicamente como la unión entre un hombre, una mujer y su progenie. Actualmente se encuentran familias uniparentales, familias ampliadas y aquellas conformadas por parejas del mismo sexo. El objetivo del presente artículo es exponer el estado de la cuestión de la investigación internacional con respecto al tema de parentalidades de familias LGBTI. Se realizó por medio de un estudio monográfico, de revisión bibliográfica actualizada. Los principales resultados son que las habilidades parentales de una persona van más allá de su orientación sexual, de ahí que las familias diversas pueden seguir un curso más normativo del que la heterosexualidad le daría crédito.

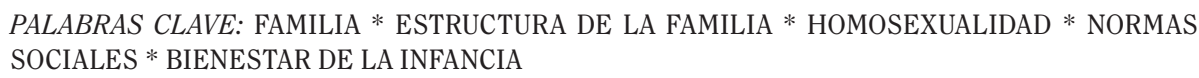

\section{ABSTRACT}

The concept of family is in transition; it's no longer the union of a women an a men and their children, now days we can find families with only one parent, extended families and those formed by same sex couples. The objective of present article is to show the state of the international research on the issue of LGBTI families. It was made by case study of current literature review. Principal results are that parenting skills of a person beyond their sexual orientation, hence the various families can follow a more normative heterosexuality which would give course credit.

KEYWORDS: FAMILY * FAMILY STRUCTURE * HOMOSEXUALITY * SOCIAL NORMS * CHILD WEL FARE

\footnotetext{
* Instituto de Investigaciones Psicológicas (IIP) de la Universidad de Costa Rica (UCR).
} tati.blancoal@gmail.com 


\section{INTRODUCCIÓN}

El presente artículo pretende aportar ideas actualizadas, basadas en evidencia, con respecto a los diferentes estilos parentales que se presentan en las parejas homosexuales que tienen hijos o hijas. Para ello es importante en primera instancia comprender la conformación de lo que se conoce como familias diversas, sus particularidades, los estilos de parentalidad utilizados y las implicaciones de tener que vivir en una sociedad basada en reglas heteronormativas.

El presente es un artículo monográfico, que se construyó por medio de la revisión bibliográfica de diversos artículos científicos, obtenidos en las diferentes bases de datos a las cuales se tiene acceso por vía electrónica. Los artículos utilizados en su mayoría cumplen el criterio de no tener más de cinco años de publicados, lo que los convierte en artículos actualizados sobre el tema a tratar.

La finalidad de exponer un tema tan polémico como el abordado en este trabajo, radica en que es importante educar a la población con respecto a las diferencias que existen en los estilos de parentalidad homosexual y heterosexual. Una vez comprendido esto, será más fácil evitar juzgar desde la heteronormatividad una realidad de muchas familias que no se rigen por las normas heterosexuales.

Se entenderá por familia diversa a aquella familia que no cumpla con la heteronormatividad, lo que significa que no se compone por una unión parental compuesta por un hombre $y$ una mujer heterosexuales, sino al contrario, por uniones hombrehombre, mujer-mujer, personas bisexuales o personas transgénero.

Las familias homoparentales son aquellas que se conforman por una pareja homosexual y los hijos o hijas que se adquieren antes o durante dicha unión. Debido a que la diversidad sexual incluye también a personas bisexuales, transexuales e intersexuales, se debe tener en cuenta que estas familias pueden ser más diversas de lo comprendido por la lógica heterosexual.

\section{TIPOS DE FAMILIAS DIVERSAS}

Una vez claro el concepto de familia diversa, es importante comprender los diferentes tipos de familias y sus estrategias de conformación, esto por el hecho de que las habilidades parentales van a ser diferentes según el tipo de familia que se conforme.

Con respecto a las posibles clasificaciones de las familias diversas, Moorefield, Pasley, Crosbie-Burnett y King (2012) describen las siguientes:

$\diamond \quad$ Las relaciones de primera cohabitación: aquellas en que dos personas se unen para conformar una familia.

$\diamond \quad$ Las de segunda cohabitación: aquellas que se conforman luego de una relación de convivencia previa.

$\diamond \quad$ Las familias diversas ensambladas: aquellas en las que existen hijos(as) de relaciones previas, o cuando una persona ahora identificada homosexual, estaba previamente involucrada en una relación heterosexual en la que se concibieron hijos.

Cuando las parejas diversas establecen matrimonios o uniones de hecho (en los países donde es un derecho constitucional) y desean tener hijos(as), podría establecerse una relación reproductiva, que consiste en una relación con otra persona con el fin de tener un hijo(a). Esto se haría por medio del acceso a espermatozoides o los vientres de alquiler con personas conocidas $y$ en otros casos por medio de la inseminación de donantes clínicamente asistida, la fertilización in vitro (FIV) o la subrogación (Dempsey, 2010).

La subrogación es una tecnología de reproducción asistida, en la que los futuros padres forjan un contrato con una mujer para llevar a sus hijos(as) en su vientre. Hay dos tipos diferentes de acuerdos de subrogación (Berkowitz, 2013):

$\diamond \quad$ Subrogación genética tradicional: cuando la madre de alquiler se implanta con el esperma de un hombre, se desarrolla el feto a término $y$ da nacimiento a un 
niño, con el que ella se relaciona genéticamente.

$\diamond \quad$ Subrogación gestacional (fertilización in vitro): se produce cuando el óvulo de otra mujer es fertilizado por uno de los espermatozoides del hombre mediante la FIV $y$ el embrión resultante se trasplanta en el útero de otra mujer.

De estos diferentes tipos de acceso a una familia, vale mencionar que por lo general los hombres tienden a elegir la subrogación, debido a que permite que alguno de los dos sea el padre biológico de los hijos; mientras que las mujeres prefieren el uso de la fecundación in vitro o la concepción asistida, donde incluso ambas pueden tener un lazo biológico con la persona menor de edad (PME), ya que una madre puede quedar embarazada de un embrión obtenido con los óvulos de su pareja (Leckey, 2011).

Tal y como señala Dempsey (2010), aquellas familias diversas en las que se concibieron hijos(as) por fecundación in vitro, inseminación artificial o subrogación, el parentesco permite explorar el significado de los lazos biológicos $y /$ genéticos para las relaciones sociales $y$ los derechos o responsabilidades de la paternidad, si es que estos vínculos son entendidos como los lazos "familiares".

Cuando se opta por la adopción, hay al menos dos maneras por las cuales se pueden conformar las familias diversas (Farr y Patterson, 2013):

$\diamond \quad$ Adopciones a extraños: describen situaciones en las que los padres biológicos no están dispuestos o son incapaces de cuidar de un niño. Se completa cuando un tribunal disuelve los vínculos jurídicos entre la PME y sus padres biológicos $y$ establece nuevos vínculos jurídicos entre la PME y sus padres adoptivos.

$\diamond \quad$ Adopciones de segundo padre: se crea el estado de paternidad legal para un segundo padre sin necesidad de terminar los derechos o responsabilidades del primer padre legal. En las familias encabezadas por parejas del mismo sexo, este tipo de adopciones crea para el niño la posibilidad de tener dos padres legalmente reconocidos.

En el caso de la adopción homoparental, es decir, que una PME pueda ser adoptada $y$ por lo tanto, se reconozca como un hijo(a) legítimo de los dos miembros de una pareja compuesta por dos personas del mismo sexo, se ha legalizado en países como España, Argentina, Bélgica, Canadá, Dinamarca, Islandia, Noruega, Reino Unido y Uruguay. Se calcula que dentro del continente norteamericano hay entre uno $y$ cinco millones de madres lesbianas, uno $y$ tres millones de padres gays, $y$ de seis a catorce millones de niños criados por padres homosexuales (Libson, 2013).

Un hallazgo interesante e importante ha sido el que las madres adoptivas lesbianas prefieren adoptar niñas a niños, siendo la motivación para esto la creencia de que los niños con dos madres van a ser más estigmatizados que las niñas, ya que no cuentan con un modelo masculino, mientras que las niñas no se verían afectadas en su feminidad (Biblarz y Savci, 2010).

Se ha considerado que los padres LGBTI son "adoptantes preferenciales", en primer lugar por el hecho de que escogen la adopción como primera vía para ser padres, lo que reduce las posibilidades de sufrir los síntomas depresivos relacionados con la interiorización del estigma de adopción. En general, los padres adoptivos LGBTI pueden mostrar algunas de las características positivas de la adopción, descritas en párrafos anteriores, que pueden beneficiar a sus hijos(as). En segundo lugar, difieren de las parejas heterosexuales en que tienen mayor voluntad para adoptar PME de un origen étnico diferente al propio (Farr y Patterson, 2013).

Las familias ensambladas homosexuales no difieren mucho a las heterosexuales en cuanto a la adaptación de los hijos(as). Tasker (2013) menciona que los hijos(as) de ambos tipos de familias reconstituidas tienen más vínculo emocional con sus padres que con su padrastro; también tienden a tener una relación más cercana con su padre no residente que con su padrastro. 
En el caso de las familias lesbianas ensambladas con hijos de familias heterosexuales previas, se ha encontrado que estas madres son más propensas que sus pares heterosexuales, a temer la pérdida de la custodia de los hijos en el proceso de divorcio. En los casos en que logran custodias compartidas o tienen la custodia total de los hijos(as), los estudios coinciden en que las PME criadas por madres lesbianas después de la separación o el divorcio heterosexual estaban también ajustadas como las criadas en otros hogares post-divorcio. En concreto, estas PME no muestran más evidencia de trastornos psicológicos, por lo general, tienen buenas relaciones con sus compañeros(as), muestran los patrones de desarrollo de género típicos y posteriormente, la mayoría se identifican como jóvenes adultos heterosexuales (Tasker, 2013).

Cuando las familias ensambladas se conforman por una pareja de lesbianas, quién se convierte en la madrastra tiende a adaptarse mejor a la vida de los hijos o hijas. Los hijos(as) pueden describir a su madrastra como una segunda madre, esto por el papel que juega en su crianza y cuidado. Hay ocasiones en que las describen como una hermana mayor o una importante amiga de la familia, esto principalmente por el hecho de que se logran acoplar a la familia, promoviendo su integración (Tasker, 2013).

Tal y como se observa en lo descrito anteriormente, las familias diversas hacen ver su diversidad no solo en el hecho de que quienes fungen como padres o madres son personas con una identidad no heterosexual, sino también por las estrategias que utilizan para poder tener una progenie. Vale mencionar que es a partir de esta conformación que se van a desarrollar o no las habilidades parentales en las parejas y por lo tanto, se debe conocer cómo se conforman las familias diversas para entender dichas habilidades.

\section{HABILIDADES PARENTALES Y PATRONES DE CRIANZA}

Anteriormente, se expuso las estrategias de conformación de las familias diversas, en donde se observa que hay diferencias si la pareja que conforma la familia es entre dos mujeres o dos hombres, esto a su vez tiene implicaciones en el desarrollo de habilidades parentales.
Por habilidad parental se va a comprender a aquella condición o habilidad que presenta una persona y que le facilita su ejercicio del rol de padre o madre. A continuación, se presentan aquellas habilidades con las que cuentan las familias diversas.

Considerando que una de las grandes habilidades parentales es la capacidad nutricia, de poder proporcionar los recursos necesarios a los hijos, es interesante el hallazgo de Regnerus (2012) quien encontró que las parejas del mismo sexo que deciden conformar una familia con hijos(as), tienden a ubicarse geográficamente en áreas urbanas y metropolitanas, 10 que les garantiza mayor acceso a recursos económicos, sociales y de salud.

Con respecto a la autopercepción de las habilidades parentales, se ha encontrado que los hombres gay pueden verse a sí mismos de forma más positiva que los hombres heterosexuales, ya que su contexto relacional no les hace ver a su pareja como el padre más calificado. Sin embargo, en el caso de las lesbianas, pueden verse a sí mismas menos positivamente que las mujeres heterosexuales, ya que están evaluando sus capacidades maternales contra una pareja femenina. Además del contexto relacional, el heterosexismo también puede afectar la habilidad percibida de las minorías sexuales (Goldberg y Smith, 2009).

Es interesante el hecho de que hay investigaciones que han demostrado que las parejas lesbianas tienen altos niveles de empleo compartido, toma de decisiones, la maternidad y el trabajo familiar al servicio de una ideología igualitaria, lo que facilita y propicia una parentalidad adecuada y evidencia la presencia de habilidades parentales en estas madres; además promedian mayor satisfacción con sus relaciones entre sí y con la crianza de los demás (Biblarz y Savci, 2010).

Cuando se compara a familias heterosexuales y familias lesbianas, se encuentra que no difieren significativamente en la implicación emocional, preocupación de los padres, la afirmación de energía, la inducción, la presencia de apoyo o el respeto a la autonomía de la PME. Sin embargo, las madres biológicas lesbianas 
presentan más establecimiento de límites en el comportamiento de la PME (Bos, 2013).

Bos (2013) reporta que las familias lesbianas con PME pequeñas son más propensas a compartir las tareas familiares que las familias de padres heterosexuales, situación que puede deberse a la ausencia de polarización de género, que lleva a compartir la carga en mayor igualdad, lo que podría explicar los hallazgos de que las madres lesbianas están más satisfechas con sus parejas en comparación con los padres heterosexuales. Las madres lesbianas están más comprometidas como madres, pasan más tiempo cuidando a sus hijos y reportan mayores niveles de implicación emocional con sus hijos(as).

Algunas diferencias entre las madres lesbianas y las madres heterosexuales, son que las primeras empiezan a pensar en tener hijos a una edad mayor que las mujeres heterosexuales; debido a que tienen que tomar varias decisiones con respecto a la concepción (por ejemplo, la decisión de escoger los donantes), misma que lleva tiempo y además, se necesita más tiempo para quedar embarazada a través de inseminación artificial que por concepción natural. Otra diferencia es que les toma más tiempo pensar en sus motivos para tener hijos(as) (Bos, 2013).

Con respecto a los patrones de crianza, Biblarz y Savci (2010) mencionan que las parejas lesbianas tienden a decorar las habitaciones de sus hijos o hijas de manera no genérica, así mismo tienden a comprar juguetes que no promuevan sexismos, lo que repercute en sus hijos(as), ya que se ha encontrado que las PME que crecen en entornos no sexistas, tienen menos actitudes estereotipadas de género. Además, mencionan que las PME entre 4 y 6 años tienden a preferir carreras acordes a su sexo, independientemente de si crecieron en una familia heterosexual o con dos madres; también reportan que las PME de entre 5 y 10 años se relacionan con su grupo de pares $y$ son aceptados por el mismo, más allá del tipo de familia que conformen.

Se ha encontrado que las familias diversas conformadas por dos hombres gay, en las que al menos uno de los miembros tuvo una relación heterosexual previa, tienden a tener estabilidad por el hecho de que experimentan un mayor nivel de calidad en la relación, porque entran en una asociación que es consistente con su identidad sexual y sus ideales de relación (Moorefield et ál., 2012).

Aunado a lo anterior, Biblarz y Savci (2010) mencionan que estos hombres tienden a presentar una co-parentalidad más equitativa, compartiendo o dividiendo el cuidado de niños(as) y las tareas domésticas de manera muy similar a las co-madres lesbianas. Además, tienen niveles más altos de utilización de técnicas de disciplina positiva y son mucho menos propensos a golpear a sus hijos(as) en comparación con los porcentajes observados en los estudios de las parejas heterosexuales $y$ sorprendentemente, incluso de co-madres lesbianas. Muchos estudios indican que cuando hay co-parentalidad entre dos hombres, lo hacen de una manera que se parece más a la de las mujeres (lesbianas y heterosexuales) que a los hombres heterosexuales casados.

En el caso de hombres gay o bisexuales, se ha encontrado que estos presentan una tendencia a querer una familia "tradicional", en la que dos personas en una relación comprometida se hacen cargo de los hijos(as) juntas y organizan su vida para ajustarse al cambio que implica tener hijos(as). Además, los acuerdos de pareja en ocasiones llevan a que uno de los padres sea quién se encargue del proceso de crianza de los hijos(as) y el otro de las responsabilidades financieras de sustento económico de la familia. Por otro lado, en cuanto a las adaptaciones que deben hacer, una de las más importantes es con respecto a sus redes de apoyo, ya que se ha visto que aquellos hombres gay o bisexuales que conforman familias, tienden a alejarse de sus círculos de amigos homosexuales, estableciendo redes de apoyo con familias heterosexuales (Power, Perlesz, McNair, Schofield, Pitts, Brown y Bickerdike, 2012).

Igualmente hay diferencias importantes entre la misma comunidad LGBTI, las posibilidades de acceso a los recursos van a diferir entre la clase media y la clase obrera, lo que puede repercutir tanto en el acceso a medios para conformar la familia, como en las habilidades 
parentales. Por ejemplo, las lesbianas blancas de clase media, tienen mayores posibilidades que las lesbianas negras de clase obrera de poder cumplir sus sueños de tener hijos(as), por las siguientes razones: a) orgullo personal por su orientación sexual; b) cuentan con redes de apoyo familiares, así como apoyo de otras madres lesbianas; c) puestos de trabajo más flexibles; d) la estabilidad financiera y e) el acceso a los médicos y las agencias de adopción. Vale destacar que el conocer a otras lesbianas que fueron madres, les da la confianza de que pueden hacer cualquier elección que quieran; pero también de que pueden acceder a los recursos que necesitan para convertirse en madres (Mezey, 2013).

Las lesbianas y los hombres gay de clase media tienen claras ventajas sobre sus contrapartes de la clase obrera, los que ocupan puestos de trabajo, no solo ganan un salario cómodo con alguna satisfacción en la carrera, sino que sus puestos de trabajo a menudo vienen con flexibilidad $y$ beneficios, incluyendo licencia por enfermedad, vacaciones y seguro de salud (Mezey, 2013).

Por tanto, según lo expuesto, se puede decir que los padres y las madres que conforman familias diversas, son personas que cuentan con las suficientes habilidades parentales, condición que las hace personas aptas para tener hijos(as) y para que estas PME sean personas funcionales durante su proceso de desarrollo. Ahora bien, el hecho de que se viva en una sociedad que sigue normas y reglas heterosexuales, impide poder validar dichas habilidades $y$ hacer efectivos los derechos a tener una familia de las personas LGBTI.

\section{HETERONORMATIVIDAD Y SUS IMPLICACIONES EN LAS FAMILIAS DIVERSAS}

Un tema más político que científico, el cual ha sido objeto de estudio, es el que se relaciona con las posibles consecuencias negativas de las PME al crecer en una familia diversa; no obstante, estudios existentes demuestran que estas diferencias no existen $y$ no hay indicios de que los padres gays $y$ madres lesbianas tengan hijos(as) inferiores, superiores o incluso, particularmente diferentes de las PME de padres heterosexuales. Al realizarse una comparación entre las PME que crecen en familias diversas con las que crecen en familias heterosexuales, los resultados muestran que en general, no hay diferencias significativas en el rendimiento escolar, la adaptación social, la salud mental, la identidad de género u orientación sexual entre los dos grupos (Hanssen, 2012).

Entre las condiciones que sí pueden afectar la conformación y estabilidad de las familias diversas, es lo que Moorefield et ál. (2012) llaman "hipótesis de institucionalización incompleta", la cual afirma que los nuevos matrimonios $y$ las familias reconstituidas experimentan más dificultades, así como, la disminución de la estabilidad relacional, debido a que sus relaciones no son compatibles con las normas sociales. Aplicado a las parejas homosexuales, ninguna de sus relaciones se institucionalizó en la mayoría de aspectos o en la mayoría de los países; para los de segunda cohabitación y las familias ensambladas, hay una doble desinstitucionalización.

Una de las razones para no aceptar socialmente las familias conformadas por hombres, es por el hecho de que este tipo de familias desafía las definiciones convencionales de la masculinidad, la paternidad y en particular, las definiciones de género, incluso, las normas sexuales dominantes de la propia cultura gay (Biblarz y Savci, 2010).

En Estados Unidos, cuando los jueces de familia deben asignar la custodia de los hijos(as) en casos de divorcio y uno de los padres es homosexual, se centran en el "interés superior del menor", en especial con lo que respecta a su desarrollo individual, tomando en cuenta aspectos como: a) la PME no debe diferir de la población en cuanto al nivel de bienestar y las relaciones entre iguales, b) la paternidad gay o lesbiana debe ser equivalente a la crianza heterosexual de los hijos(as) y c) el contacto permanente con los padres heterosexuales de sexo contrario a la PME debe ocurrir (Tasker, 2013).

Con respecto a la influencia de la religión, tema que al menos en Costa Rica es de gran importancia, Lytle, Foley y Aster (2013) encontraron que la intolerancia que promueven muchas veces diferentes grupos religiosos, 
podría traer consecuencias negativas para estas familias, llevando a la internalización de la homonegatividad; no obstante, si se logra resolver el aparente conflicto entre religión y diversidad sexual, las familias se pueden ver fortalecidas.

En relación al tema de la religión, cabe decir que en América Latina la influencia de la Iglesia Católica es tanta que cualquier intento de resistir a los principios católicos es considerado por diversos sectores como un ataque contra el Estado. El patriarcado y la heteronormatividad de la iglesia son vistos como naturales y legítimos. Por otra parte, muchas de las características de la maternidad están incrustadas en el modelo católico de la Virgen María; por ejemplo, que una madre se sacrifique en nombre de sus hijos y muestre la sensibilidad $y$ el cuidado de estos (Lubbe, 2013).

Como consecuencia de la falta de legitimidad social y legal de las familias diversas, las madres no biológicas podrían sentirse excluidas en su papel de madres por parte de las instituciones. Además de experimentar una mayor sensación de exclusión, las madres no biológicas pueden experimentar falta de reconocimiento, de derecho $y$ de seguridad en su rol parental (Bos, 2013).

En Costa Rica, tal y como señala Redondo (2006), los requisitos para la adopción se establecen en el art. 106 del Código de Familia:

a) Poseer capacidad plena para ejercer sus derechos civiles.

b) Ser mayor de veinticinco años, en caso de adopciones individuales. En adopciones conjuntas, bastará que uno de los adoptantes haya alcanzado esta edad.

c) Ser por lo menos quince años mayor que el adoptado. En la adopción conjunta, esa diferencia se establecerá con respecto al adoptante de menor edad. En la adopción por un solo cónyuge, esa diferencia también deberá existir con el consorte del adoptante.

d) Ser de buena conducta y reputación. Estas cualidades se comprobarán con una prueba idónea, documental o testimonial, que será apreciada y valorada por el Juez en sentencia. e) Poseer condiciones familiares, morales, psicológicas, sociales, económicas y de salud, que evidencien aptitud y disposición para asumir la responsabilidad parental.

En estos no está establecido como requisito ser heterosexual, por lo que no permitir la adopción a personas homosexuales, ya sea que se encuentren en convivencia con una pareja o se encuentran solteras, es una evidencia de discriminación por orientación sexual.

En aquellos casos en que los hijos(as) de familias diversas han sido estigmatizados, tres grupos muestran una mayor capacidad de recuperación; es decir, las PME que asisten a escuelas que tienen programas de concientización de diversidad sexual en sus planes de estudio, las PME cuyos padres se describen a sí mismos como miembros activos de la comunidad LGBTI y las PME que tienen contacto frecuente con otros hijos(as) de padres del mismo sexo (Bos, 2013).

Así como hay personas LGBTI que desean tener hijos(as), hay quienes no poseen el mismo deseo. Riskind y Patterson (2010) explican que estas personas son menos propensas a tener hijos que sus contrapartes heterosexuales $y$ exponen como posibles causas: a) la falta de deseo de convertirse en padres $y$ b) la falta de intención de convertirse en padres. Los deseos consisten en lo que uno quiere o le gustaría hacer, mientras que las intenciones consisten en lo que uno se propone o planea hacer. Situación que es diferente para hombres y mujeres, ya que los hombres gay sin hijos(as) tienen menos probabilidades que sus pares heterosexuales de expresar los deseos e intenciones de paternidad. Las mujeres lesbianas sin hijos(as) tienen menos probabilidades que sus pares heterosexuales de expresar los deseos de maternidad; aunque las mismas probabilidades que sus pares heterosexuales de expresar las intenciones de ser madres.

Una manera de unir a las familias diversas es combatiendo la homonegatividad y el heterosexismo, apoyando la igualdad de derechos de las personas gay, lesbianas, bisexuales y transexuales (LGBTI), además de que se ha estudiado el impacto que dichos mensajes puedan 
tener en el desarrollo de las personas menores de edad $y$ no se ha encontrado que influyan negativamente en su ajuste personal o familiar (Lytle, Foley y Aster, 2013).

Con el fin de contrarrestar la heteronormatividad y cómo esta influye en los procesos legales de adopción y otras maneras de conformar una familia, Hanssen (2012) menciona que algunos estudios proponen una divulgación temprana de la orientación sexual de los padres para contrarrestar el estigma social. También indica que la apertura puede contribuir a nuevas representaciones de la familia y la paternidad, desafiando la heteronormatividad dominante que afirma que los padres heterosexuales son los guardianes preferidos.

Por último, se destaca que velar por el interés superior de la persona menor de edad, implica que esta va contar con una familia conformada por padres, cuyas habilidades parentales son las suficientes como para que las PME lleguen a ser personas funcionales socialmente.

A lo largo del trabajo se estableció que las familias diversas por lo general están formadas por dos personas adultas que desarrollan habilidades parentales que facilitan su rol paterno o materno. Por lo tanto, a razón de existir un problema con estas familias, este se debe a la heteronormatividad desde la cual son analizadas y no al tipo de familia que conforman.

\section{CONCLUSIONES}

El presente artículo expuso el tema de los estilos de parentalidad que se presentan en las familias diversas y cómo estas se ven afectadas por el contexto heteronormativo.

Las habilidades parentales de una persona van más allá de su orientación sexual, de ahí que las familias diversas pueden seguir un curso más normativo del que la heterosexualidad le daría crédito.

Se espera que al brindar aportes basados en evidencia, quienes tengan acceso a la información planteada, logren ampliar su perspectiva sobre el tema, favoreciendo la convivencia entre las familias, más allá de cómo estas se conforman, evitando caer en juicios de valor, promovidos por la lógica heterosexual predominante.

$\mathrm{El}$ acceso a la familia es un Derecho Humano y por lo tanto, se debe garantizar a todas las personas, independientemente de su orientación e identidad sexual. Las implicaciones legales de denegar un Derecho Humano no son más amplias, simplemente por el hecho de que las leyes, al menos en Costa Rica, están basadas en la lógica confesional y en la moral religiosa, por lo tanto, son pocas las personas dispuestas a cambiarlas.

Es importante estudiar este tema en nuestra realidad nacional, ya que de esta manera se podría presentar evidencia desde nuestro contexto, que facilite el poder garantizar desde lo legal y lo político, el Derecho Humano de tener una familia, así como para facilitar la adopción a las personas LGBTI.

\section{BIBLIOGRAFÍA}

LIBROS

Berkowitz, Dana. "Gay men and surrogacy". Chapter II. LGBT-Parent families: innovations in research and implications for practice. Abbie Goldberg y Katherine Allen (eds.). Inglaterra. Springer New York Heidelberg Dordrecht London, 2013: 71-86.

Bos, Henny. "Lesbian-mother families formed through donor insemination". Chapter I. LGBT-Parent families: innovations in research and implications for practice. Abbie Goldberg y Katherine Allen (eds.) Inglaterra. Springer New York Heidelberg Dordrecht London, 2013: 21-38.

Farr, Rachel y Patterson, Charlotte. "Lesbian and gay adoptive parents and their children". Chapter I. LGBT-Parent families: innovations in research and implications for practice. Abbie Goldberg y Katherine Allen (eds.). Inglaterra. Springer New York Heidelberg Dordrecht London, 2013: 39-58. 
Lubbe, Carien. "LGBT parents and their children: non-western research and perspectives". Chapter II. LGBT-Parent families: innovations in research and implications for practice practice. Abbie Goldberg y Katherine Allen (eds.). Inglaterra. Springer New York Heidelberg Dordrecht London, 2013: 209-224.

Mezey, Nancy. "How lesbians and gay men decide to become parents or remain childfree". Chapter II. LGBT-Parent families: innovations in research and implications for practice. Abbie Goldberg y Katherine Allen (eds.). Inglaterra. Springer New York Heidelberg Dordrecht London, 2013: 59-70.

Tasker, Fiona. "Lesbian and gay parenting postheterosexual divorce and separation". Chapter I. LGBT-Parent families: innovations in research and implications for practice. Abbie Goldberg y Katherine Allen (eds.). Inglaterra. Springer New York Heidelberg Dordrecht London, 2013: 3-20.

\section{PUBLICACIONES PERIÓDICAS}

Biblarz, Timothy y Savci, Evren. "Lesbian, gay, bisexual, and transgender families". Journal of Marriage and Family 72. Estados Unidos. National Council on Family Relations, junio, 2010: 480-497.

Dempsey, Deborah. "Conceiving and negotiating reproductive relationships: lesbians and gay men forming families with children". Sociology 44. Reino Unido. British Sociological Association-SAGE Publications Ltd., 2010: 1145-1162.

Goldberg, Abbie y Smith, JuliAnna. "Perceived parenting skill across the transition to adoptive parenthood among lesbian, gay, and heterosexual couples". Journal of Family Psychology 23 (6). Washington DC, Estados Unidos. American Psychological Association, diciembre, 2009: 861-870.
Hanssen, Jorid. "My rainbow family': discomfort and the heteronormative logics". Young 20 (3). Washington DC, Estados Unidos. SAGE Publications and YounG Editorial Group, agosto, 2012: 237-256.

Leckey, Robert. "Law reform, lesbian parenting, and the reflective claim". Social \& Legal Studies 20 (3). SAGE. Setiembre, 2011: 331-348.

Libson, Micaela. "Parentalidades gays y lesbianas: el surgimiento de la temática en Argentina". Revista de Ciências Sociais 44 (1). Fortaleza, Brasil. Departamento de Ciências SociaisUniversidade Federal do Ceará (UFC). Enero-junio, 2013: 109-131. En: <http:// www.rcs.ufc.br/edicoes/ $v 44 \mathrm{n} 1 / \mathrm{rcs}_{-}$ v44n1a5.pdf $>$ [consultado el 3 de julio de 2014].

Lytle, Megan; Foley, Pamela y Aster, Amanda. "Adult children of gay and lesbian parents: religion and the parentchild relationship". The Counseling Psychologist 41 (4). Washington DC, Estados Unidos. American Psychological Association, 2013: 530-567.

Moorefield, Brad; Pasley, Kay; Crosbie-Burnett, Margaret y King, Erin. "Explaining couple cohesion in different types of gay families". Journal of Family Issues 2 (33). Washington DC, Estados Unidos. American Psychological Association, 2012: $182-201$.

Power, J.; Perlsz, A.; McNair, R.; Schofield, M.; Pitts, M.; Brown, R. y Bickerdike, A. "Gay and bisexual dads and diversity: fathers in the work, love, play study". Journal of Family Studies 18(2-3). SAGE Publications, 2012: 143-154. En: <http:// mra.econtentmanagement.com/archives/ vol/7/issue/3/call/> [consultado el 3 de julio de 2014].

Regnerus, M. "How different are the adult children of parents who have same-sex relationships? Findings from the new family structures study". Social Science Research 41. España. Elsevier B.V. 
Julio, 2012: 752-770. En: <http://dx.doi. org/10.1016/j.ssresearch.2012.03.009> [consultado el 3 de julio de 2014].

Riskind, R. y Patterson, C. "Parenting intentions and desires among childless lesbian, gay, and heterosexual individuals". Journal of Family Psychology 24 (1). Washington DC, Estados Unidos. American Psychological Association, 2010: 78-81.

Van Eeden-Moorefield, B.; Pasley, K.; CrosbieBurnett, M. y King, E. "Explaining couple cohesion in different types of gay families". Journal of Family Issues 33 (2). SAGE Publications. Febrero, 2012: 182-201.

\section{TESIS}

Redondo, L. "La adopción por parte de parejas o personas homosexuales: un desafío en el reconocimiento de los derechos humanos". [Trabajo Final de Graduación para optar por el Grado de Máster en Derechos Humanos]. Universidad Estatal a Distancia, Sede de Sabanilla de Montes de Oca, 2006.

Fecha de ingreso: $27 / 07 / 2014$

Fecha de aprobación: 30/10/2014 